\title{
Elevada porcentagem de blastocistose em escolares de São Paulo, SP
}

\author{
Blastocystosis: a high proportion of cases found in schoolchildren of \\ São Paulo, State of São Paulo, Brazil
Vicente Amato Neto ${ }^{1}$, Ruth Semira Rodríguez Alarcon ${ }^{1}$, Erika Gakiya ${ }^{1}$, Cláudio Santos Ferreira ${ }^{1}$, Rita Cristina Bezerra ${ }^{1}$ e Alexsander Gonzaga dos Santos ${ }^{1}$

\section{RESUM0}

Em exame parasitológico de fezes de 227 alunos de escola pública de São Paulo ( SP), encontramos 87 (38,3\%) positivas para Blastocystis hominis. A blastocistose ainda suscita controvérsias e indefinições, merecedoras de esclarecimentos sobretudo para evitar contratempos no âmbito da saúde pública e das atenções médico-assistenciais.

Palavras-chaves: Blastocystis hominis. Blastocistose. Escolares. São Paulo. Elevada porcentagem. Controvérsias e indefinições.

\begin{abstract}
As a part of medical assistance activities, parasitological examination of fecal samples from 227 school children from a public institution of São Paulo (SP) revealed a rather high proportion of results positive for Blastocystis hominis. Other protozoan and worm species were markedly scarcer, a peculiar situation according to our judgement. It is acknowledged that blastocystosis is still largely an indefinite and controversial subject, which deserves adequate analysis to avoid drawbacks in the sphere of action of public health and general medical assistance.
\end{abstract}

Key-words: Blastocystis hominis. Blastocystosis. School children. São Paulo. High percentages. Controversy.

Blastocystis hominis é protozoário freqüentemente encontrado em exame parasitológico de fezes. Estão relacionadas com ele várias controvérsias e indefinições, sobretudo referentes à epidemiologia, à transmissão, aos aspectos clínicos e à patogenicidade. Acerca do diagnóstico também são vigentes algumas situações merecedoras de melhores posicionamentos e divulgação ${ }^{1}$.

Ao realizarmos análise de fezes de crianças, em atividade assistencial, encontramos expressiva porcentagem de positividades concernentes ao B. hominis e essa circunstância estimulou-nos a efetuar comentários que têm nexo com a participação plena de dúvidas de tal microrganismo.

Pareceu-nos importante abordar questões vinculadas à blastocistose porque é necessário conhecer particularidades provavelmente úteis para entendê-la melhor porquanto impõe-se minimizar as incertezas existentes e auxiliar de forma correta tarefas diagnósticas ou clínico-terapêuticas.

Examinamos, desde 7 de outubro até 15 de dezembro de 2003, as fezes de 227 alunos da escola estadual "Oswaldo Valder", situada no bairro denominado Boa Vista, que está localizado na periferia do Município de São Paulo. As idades variaram de seis a dez anos. 0 padrão de vida pôde ser qualificado como baixo.

As matérias fecais foram colocadas em recipiente que faz parte do sistema "Coprotest", comercializado no Brasil.

Sempre recorremos a três métodos: direto; da centrífugoflutuação em solução de sulfato de zinco (Faust e cols); da sedimentação espontânea em água ( Lutz) ${ }^{2}$.

1. Laboratório de Investigação Médica Parasitologia do Departamento de Moléstias Infecciosas e Parasitárias do Hospital das Clínicas da Faculdade de Medicina da Universidade de São Paulo, São Paulo, SP.

Endereço para correspondência: Prof. Vicente Amato Neto. Laboratório de Investigação Médica-Parasitologia/DMI/HC/FM/USP. Av. Dr. Enéas de Carvalho Aguiar 470, 05403-000 São Paulo, SP.

Tel: 11 3066-7042, Fax: 11 3081-8144

e-mail:amatonet@usp.br

Rebido para publicação em 8/4/2004

Aceito em 21/5/2004 
Os resultados, em números e porcentagens, encontramse a seguir especificados:

Blastocystis hominis ( $87-38,3 \%)$;
Entamoeba coli ( $30-13,2 \%)$
Endolimax nana (22-9,7\%)
Giardia lamblia (12-5,3\%);
Entamoeba histolytica ou díspar (6 - 2,6\%);
Trichuris trichiura ( $-1,3 \%)$;
Ascaris lumbricoides ( $2-0,9 \%)$;
Hymenolepis nana $(2-0,9 \%)$;
Enterobius vermicularis (1 - 0,4\%).

Quanto ao B. hominis_computamos alguns detalhamentos: positividade no direto - 75 (33\%); no de Fauste cols - 48 (21,1\%); no de Lutz - 74 (32,6\%). Ainda segundo as técnicas, o B. hominis ficou revelado exclusivamente pelo direto $9(3,9 \%)$ vezes, pelo de Fauste cols 3 (1,3\%) e pelo de Lutz 7 (3,1\%). Em 55 (22,2\%) exames não detectamos associação do B. hominis com outros protozoários ou ovos de helmintos encontrados.

Aporcentagem pertinente ao B. hominis compareceu de forma extremamente expressiva e predominante, suscitando especulações. As taxas de presenças de ovos de helmintos mostraram-se, ao contrário, surpreendentemente baixas se lembrarmos que as crianças residem em uma mesma região, evidentemente com condições sanitárias iguais, sem terem sido tratadas com antihelmínticos mediante programas abrangentes e repetitivos.

Agimos somente como laboratoristas e, então, não contamos com informações de caráter clínico.

Blastocistose éainda conceituada ou interpretada de maneiras heterogêneas ou inadequadas ${ }^{1}$. Na imensa maioria de publicações que têm como autores profissionais respeitados $0 \mathrm{~B}$. hominis não é citado nas especificações de resultados obtidos em inquéritos e destinados a avaliar a presença de protozoários e helmintos em diferentes grupos de pessoas, representadas por crianças ou

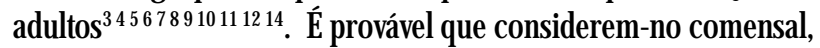
mas paradoxalmente incluem o Chilomastix mesnili, a Endolimax nana, a Entamoeba coli e a Iodamoeba bütschlii, sem dúvida, pelo menos presentemente, tidos como comensais. Ainda mais, nos títulos dos artigos e de tabelas que citam esses protozoários referem-se a parasitas intestinais ${ }^{347911} 131415$.

Determinados observadores, cremos, não sabem reconhecer e identificar o B. hominis ou, simplesmente, não concedem qualquer importância a ele, decidindo não mencioná-lo nos resultados dos exames. Em laboratório visitado por um de nós ( VAN) afirmaram que o encontro desse protozoário significa que as fezes são velhas e mal conservadas.

0 diagnóstico requer conjecturas. 0 simples processo direto, executado com soluções salina e de Lugol, é prestimoso. Preparações permanentes, exemplificadas pelas colorações por meio de hematoxilina férrica, igualmente afiguram-se convenientes. Porém, água e diversas soluções lisam 0 protozoário, decorrendo disso resultados falsos negativos.
Mencionamos que água e soluções podem desintegrar 0 B.hominis conturbando os diagnósticos por determinadas técnicas. Porém, no relato de resultados que obtivemos vê-se que os processos de Fauste cols e de Lutz evidenciaram bons desempenhos. Entretanto, possuímos adequada explicação. As fezes remetidas para análise foram coletadas em recipiente do sistema citado, que contém formol a 10\% tamponado como conservante.

Năo está categoricamente provado que 0 B. hominisé comensal como admitem várias pessoas, inclusive em tarefas médicoassistenciais. Por outro lado, há os que consideram a possibilidade dele causar manifestações clínicas, sobretudo quando excluídos outros agentes tido como patogênicos. Outrossim, julgam que a quantidade encontrada influi, em especial nos indivíduos imunodeprimidos. Năo se pode ainda apontar com segurança a maneira de quantificar, comumente derivada de simples exame microscópico. Conhecemos médicos que indicam tratamento se com aumento de quatrocentas vezes aparecem cinco ou mais exemplares por campo.

As controvérsias e indefinições que persistem a respeito da blastocistose precisam ser superadas. A parasitologistas, infectologistas, gastrenterologistas, epidemiologistas e sanitaristas, por exemplo, cabe a tarefa de elucidar as dúvidas ou, pelo menos, de atenuá-las, a fim de evitar contratempos no âmbito da saúde pública e das atenções médicoassistenciais $^{3916}$.

\section{REFEÊNCIAS BIBLIOGRÁFICAS}

1. Amato Neto V, Alarcón RSR, Gakiya E, Bezerra RC, Ferreira CS, Braz LMA. Blastocistose: controvérsias e indefinições. Revista da Sociedade Brasileira de Medicina Tropical 36:515-517, 2003.

2. Amato Neto V, Corrêa LL. Exame parasitológico das fezes. Sarvier, São Paulo, 1991.

3. Branco Junior HC, Rodrigues JC. Importância de aspectos sanitários e educacionais na epidemiologia de enteroparasitoses em ambientes rurais. Revista Brasileira de Análises Clínicas 31:87-90, 1999.

4. Cantos GA, Dutra RL, Hilsendeger T, Guidis ARG. Análise quanto a ocorrência de parasitas intestinais em amostras fecais processadas em um laboratório de Criciúma-SC. NewsLab 56:78-86, 2003.

5. Costa-Macedo LM, Rey L. Frequency and precocity of human intestinal parasitism in a group of infants from Rio de Janeiro, Brazil. Revista do Instituto de Medicina Tropical de São Paulo 39:305-306, 1997.

6. Ferreira CS, Ferreira MU, Nogueira MR. The prevalence of infection by intestinal parasites in an urban slum in São Paulo, Brazil. Journal of Tropical Medicine and Hygiene 97:121-127, 1994.

7. Fontes G, Oliveira KKL, Oliveira AKL, Rocha EMM. Influência do tratamento específico na prevalência de enteroparasitoses e esquistossomose mansônica em escolares do município de Barra de Santo Antonio, AL. Revista da Sociedade Brasileira de Medicina Tropical 36:625-628, 2003.

8. Giazzi JF, Martini AS, Buainain A, Mendonça CP, Belda Neto M, Santos JL. Levantamento de protozoários e helmintos em crianças de um núcleo populacional de Araraquara. Revista Brasileira de Farmácia 43: 49-52, 1982.

9. Ludwig KM, Frei F, Álvares Filho F, Ribeiro-Paes JT. Correlação entre condições de saneamento básico e parasitoses intestinais na população de Assis, Estado de São Paulo. Revista da Sociedade Brasileira de Medicina Tropical 32:547-555, 1999.

10. Martini AS, Rodrigues VC, Taba MRM, Fujimori C. Avaliação da presença de enteroparasitas em crianças de um Centro de Convivência Infantil. Revista da Faculdade de Odontologia de Ribeirão Preto 22:17-20, 1985. 
11. Oliveira MR, Barbosa MA, Salata E, Sogayar MITL, Sogayar R, Corrêa FMA. Prevalência de enteroparasitas na população urbana do 20 Subdistrito de Botucatu. Revista de Saúde Pública 8:213-234, 1974.

12. Salata E, Corrêa FMA, Sogayar R, Sogayar MIL, Barbosa MA. Inquérito parasitológico na CECAP - distrito-sede de Botucatu, Estado de São Paulo, Brasil. Revista de Saúde Pública 6:385-392, 1972.

13. Santos DE, Wiebbelling AMP, Mezzari A. Parasitos intestinais: aspectos gerais e prevalência em uma escola da periferia de Porto Alegre-RS. NewsLab 60:118-134, 2003.

14. Silva CG, Santos HA. Ocorrência de parasitoses intestinais da área de abrangência do Centro de Saúde Cícero Ildefonso da Regional Oeste da
Prefeitura Municipal de Belo Horizonte, Minas Gerais. Revista de Biologia e Ciências da Terra 1:1-11, 2001.

15. Tavares-Dias M, Grandini AA. Prevalência e aspecto epidemiológicos de enteroparasitoses na população de São José da Bela Vista, São Paulo. Revista da Sociedade Brasileira de Medicina Tropical 32:6365, 1999.

16. Vinha C. Incidência, no Brasil, de helmintos transmitidos pelo soloRotina coproscópica do ex-Departamento Nacional de endemias rurais. Apresentado no XVIII Congresso Brasileiro de Higiene, São Paulo, 1970. 